\author{
Dimitry V. Balandin \\ Research Institute for Applied \\ Mathematics and Cybernetics \\ Nizhni Novgorod State University \\ N.I. Lobatchevsky \\ 10 Uljanova Str. \\ Nizhni Novgorod, 603005, Russia
}

\title{
On Maximum Energy of Mechanical Systems Under Shock Disturbances
}

\begin{abstract}
In this article, we develop a method for estimating the maximum energy of a mechanical system subjected to disturbances whose integral absolute value is bounded. The conditions for this estimate to be exact are found. The method is demonstrated by calculating the maximal displacement in a second-order nonlinear system. (C) 1993 John Wiley \& Sons, Inc.
\end{abstract}

\section{INTRODUCTION}

When designing and developing different engineering systems we often encounter the problem of determining the maximum response of the system under disturbances of some specified class. One of the first problems solved is the wellknown Boulgakov's problem of accumulating disturbances in linear systems [Boulgakov, 1946]. In this problem, the absolute values of the external disturbances are supposed to have a bounded integral over time. A number of papers [Balandin, 1989; Bolotnik, 1976; Sevin, 1957] deal with the maximum displacement in systems under disturbances, the integral of whose absolute value is bounded. In Balandin [1989], a broad class of nonlinear second-order systems is found in which the maximum displacement is reached at a single instantaneous impact. In this paper, we consider the problem of estimating the maximal energy of mechanical systems subjected to a disturbance whose absolute value is integrable over time. If such a disturbance is sign-definite, it can be physically interpreted as a force is with a finite impulse.

\section{MECHANICAL SYSTEM AND STATEMENT OF PROBLEM}

Let us consider a mechanical system consisting of $N$ point masses, $P_{\nu}$, connected to a rigid body

Shock and Vibration, Vol. 1, No. 2, pp. 135-144 (1993) (C) 1993 John Wiley \& Sons, Inc. (called a base) moving without rotation in an inertial reference system. Denote by $S_{o}$ a reference system rigidly connected to the base. Let $\mathbf{r}_{\nu}$ be the position vectors of the point masses in the system $S_{o}$. Now for this mechanical system let us introduce the generalized coordinates $q_{1}, q_{2}$, $\ldots, q_{n}$ describing the motion of the system with respect to the base. Because our system is scleronomous, position vectors $\mathbf{r}_{\nu}$ of points $\boldsymbol{P}_{\nu}$ do not depend on time explicitly and can be expressed as functions of $q_{1}, q_{2}, \ldots, q_{n}$ :

$$
\mathbf{r}_{\nu}=\mathbf{r}_{\nu}\left(q_{1}, q_{2}, \ldots, q_{n}\right)
$$

If the mechanical system is holonomic, then its motion can be described by Lagrange equations [Pars, 1968]

$$
\frac{d}{d t} \frac{\partial L}{\partial \dot{q}_{i}}-\frac{\partial L}{\partial q_{i}}=Q_{i}
$$

with initial conditions $q_{i}(0)=q_{i}^{o}, \dot{q}_{i}(0)=\dot{q}_{i}^{o}$, where $L=T-\Pi$ is a Lagrange function, $T$ and $\Pi$ are the kinetic and potential energies, respectively, and $Q_{i}$ are the generalized forces. The generalized forces may be described as a sum of dissipative $\left(Q_{i}^{d}\right)$, gyroscopic $\left(Q_{i}^{g}\right)$, and inertia $\left(Q_{i}^{m}\right)$ forces:

$$
Q_{i}=Q_{i}^{d}+Q_{i}^{g}+Q_{i}^{m}
$$

CCC 1070-9622/93/020135-10 
Note that for dissipative and gyroscopic forces the conditions

$$
\sum_{i=1}^{n} Q_{i}^{d} \dot{q}_{i} \leq 0, \sum_{i=1}^{n} Q_{i}^{g} \dot{q}_{i}=0
$$

hold.

The virtual work of inertia forces is given by

$$
-\sum_{\nu=1}^{N} m_{\nu} \delta \mathbf{r}_{\nu} \mathbf{w}(t)=-M \delta \mathbf{R}_{c} \mathbf{w}(t)=\sum_{i=1}^{n} Q_{i}^{m} \delta q_{i}
$$

where $m_{\nu}, \delta \mathbf{r}_{\nu}$ are the mass and virtual displacement of the point $P_{\nu} ; M=\sum_{\nu=1}^{N} m_{\nu}$ is the total mass of the system; $\mathbf{R}_{c}=1 / M \sum_{\nu=1}^{N} m_{\nu} \mathbf{r}_{\nu}$ is the position vector of the system mass center; $\delta \mathbf{R}_{c}$ is the virtual displacement of the mass center; $\mathbf{w}(t)$ is the acceleration of the base expressed in the reference system $S_{o} ; \delta q_{i}$ is the virtual displacement of the generalized coordinate $q_{i}$. Because our system is scleronomous, the following equation is valid for inertia forces

$$
\sum_{i=1}^{n} Q_{i}^{m} \dot{q}_{i}=-M \dot{\mathbf{R}}_{c} \mathbf{w}(t)
$$

Let us now proceed to the problem statement. Suppose that the vector function $\mathbf{w}(t)$ describing an external disturbance acting on the system is piecewise continuous and belongs to the class of so-called shock disturbances

$$
\begin{aligned}
\Theta= & \{\mathbf{w}(\cdot): \mathbf{w}(t) \equiv 0 \\
& \text { for } \left.t<0, \int_{o}^{\infty}|\mathbf{w}(t)| d t \leq J_{o}\right\} .
\end{aligned}
$$

For each function of the above class, a mechanical system response will be understood as a maximal (over time) sum of kinetic and potential energies. This sum is obtained by solving system (1) with the specified vector $\mathbf{w}(t)$ :

$$
W[\mathbf{w}(\cdot)]=\sup _{t \in[o, \infty)}(T+\Pi) .
$$

Additionally, the potential energy as a function of generalized coordinates

$$
\Pi=\Pi\left(q_{1}, q_{2}, \ldots, q_{n}\right) \geq 0
$$

is assumed nonnegative.
Let us state the following problem: find the estimate from above for the maximum of the total energy, that is, determine the number $W^{+}$so that

$$
W[\mathbf{w}(\cdot)] \leq W^{+} \quad \forall \mathbf{w}(\cdot) \in \Theta .
$$

\section{ANALYSIS OF THE PROBLEM}

As it is known [Pars, 1968], the rate of change of the energy $E=T+\Pi$ for a holonomic system is given by

$$
\frac{d E}{d t}=\sum_{i=1}^{n} Q_{i} \dot{q}_{i}
$$

Invoking (2) and (3), we rewrite this equation as

$$
\frac{d E}{d t}=\sum_{i=1}^{n} Q_{i}^{d} \dot{q}_{i}-M \dot{\mathbf{R}}_{c} \mathbf{w}(t) .
$$

Now, let us take the time segments $\Omega_{k}=\left[t_{k}^{-}, t_{k}^{+}\right]$, within which we have

$$
\frac{d E}{d t} \geq 0,
$$

and denote the union of these segments by

$$
\Omega_{*}=\bigcup_{k} \Omega_{k} .
$$

Taking into account the inequality in (2), we can prove the inequalities

$$
\begin{gathered}
\sum_{i=1}^{n} Q_{i}^{d} \dot{q}_{i}-M \dot{\mathbf{R}}_{c} \mathbf{w}(t) \geq 0, \\
\frac{d E}{d t} \leq\left|M \dot{\mathbf{R}}_{c} \mathbf{w}(t)\right|
\end{gathered}
$$

to hold for all $t \in \Omega_{*}$. Note that the kinetic energy of a point mass $M$ moving at the same speed as the system mass center does not exceed the kinetic energy of the whole system:

$$
\frac{M \dot{\mathbf{R}}_{c}^{2}}{2} \leq T=\sum_{\nu=1}^{N} \frac{m_{\nu} \dot{\mathbf{r}}_{\nu}^{2}}{2} .
$$

Besides, because the potential energy is assumed nonnegative (5), we have

$$
\frac{M \dot{\mathbf{R}}_{c}^{2}}{2} \leq E,
$$


and hence

$$
\left|\dot{\mathbf{R}}_{c}\right| \leq \sqrt{\frac{2 E}{M}} .
$$

Therefore,

$$
\frac{d E}{d t} \leq \sqrt{2 M E}|\mathbf{w}(t)| .
$$

By integrating the last inequality over the arbitrary segment $\Omega_{k}$ we obtain

$$
\sqrt{E_{k}^{+}}-\sqrt{E_{k}^{-}} \leq \sqrt{M / 2} \int_{t_{\bar{k}}}^{t_{\bar{k}}^{+}}|\mathbf{w}(t)| d t,
$$

where $E_{k}^{-}=E\left(t_{k}^{-}\right), E_{k}^{+}=E\left(t_{k}^{+}\right)$. Adding all the inequalities obtained for all segments $\Omega_{k}$ and taking Eq. (4) into account, we arrive at

$$
\begin{aligned}
\sum_{k=1}^{L}\left[\sqrt{E_{k}^{+}}-\sqrt{E_{k}^{-}}\right] & \leq \sqrt{M / 2} \sum_{k=1}^{L} \int_{t_{\bar{k}}}^{t_{k}^{+}}|\mathbf{w}(t)| d t \\
& \leq \sqrt{M / 2} J_{o} .
\end{aligned}
$$

According to the definition of the segments $\Omega_{k}$, we have

$$
E_{L}^{+}=\sup _{t \in[o, \infty)} E(t)=W[\mathbf{w}(\cdot)] .
$$

Note that $E_{k+1}^{-} \leq E_{k}^{+}$, and hence,

$$
\sqrt{E_{L}^{+}}-\sqrt{E_{1}^{-}} \leq \sum_{k=1}^{L}\left[\sqrt{E_{k}^{+}}-\sqrt{E_{k}^{-}}\right] .
$$

Finally we obtain

$$
\sqrt{E_{L}^{+}} \leq \sqrt{E_{1}^{-}}+\sqrt{M / 2} J_{o}
$$

or

$$
E_{L}^{+}=W[\mathbf{w}(\cdot)] \leq\left\{\sqrt{E_{1}^{-}}+\sqrt{M / 2} J_{o}\right\}^{2} .
$$

Here, $E_{1}^{-}$is determined by initial conditions for Eq. (1) and is just the value of the system energy at the instant when the disturbance starts to act

$$
W^{+}=\left\{\sqrt{E_{1}^{-}}+\sqrt{M / 2} J_{o}\right\}^{2} .
$$

\section{DISCUSSION}

The above estimate for maximum energy of a system under shock disturbances gives rise to a quite natural question whether there exist mechanical systems for which this estimate is exact, that is, whether there exist systems for which

$$
W^{+}=\sup _{\mathbf{w}(\cdot) \in \Theta} W[\mathbf{w}(\cdot)]
$$

is valid. Before formulating validity conditions for (8), let us estimate a system response to the instantaneous impact given by

$$
\mathbf{w}(t)=J_{o} \mathbf{e} \delta(t),
$$

where $\mathbf{e}$ is a constant unit vector $\left(\mathbf{e}^{2}=1\right) ; J_{o}$ is a positive number; and $\delta(t)$ is the Dirac delta-function for which

$$
\int_{-\infty}^{\infty} \delta(t) d t=1 .
$$

It is known [Pars, 1968] that the instantaneous impact (9) results in an instantaneous change in the velocities $\dot{\mathbf{r}}_{\nu}$ of the points $\boldsymbol{P}_{\nu}$ by the vectors $\mathrm{c}_{\nu}$, with the following inequality holding

$$
c_{\nu}^{2} \leq J_{o}^{2}
$$

Let us denote by $T_{c}\left(J_{o}\right)$ the magnitude by which the kinetic energy of the system mass center is changed under instantaneous impact (9). From (10), we obtain

$$
T_{c}\left(J_{o}\right) \leq \frac{M J_{o}^{2}}{2} .
$$

Now let us state the conditions under which the equality (8) holds. Let the initial conditions in (1) be such that

$$
\begin{gathered}
\dot{q}_{1}^{o}=\dot{q}_{2}^{o}=\cdots=\dot{q}_{n}^{o}=0, \\
\Pi\left(q_{1}^{o}, q_{2}^{o}, \ldots, q_{n}^{o}\right)=0,
\end{gathered}
$$

and let the unit vector e be such that the change in the mass center kinetic energy due to the instantaneous impact (9) is

$$
T_{c}\left(J_{o}\right)=\frac{M J_{o}^{2}}{2} .
$$

Then, equality (8) is true. Indeed, for any disturbance $\mathbf{w}(\cdot) \in \Theta$, taking (11) into account, we obtain $E_{1}^{-}=0$, and hence 


$$
W^{+}=\frac{M J_{o}^{2}}{2} \text {. }
$$

On the other hand, when taking the disturbance

$$
\mathbf{w}^{*}(t)=J_{o} \mathbf{e}_{\nu}(t)
$$

where the scalar function $\nu(t)$ is determined by

$$
\nu(t)= \begin{cases}1 / \tau, & 0 \leq t \leq \tau \\ 0, & t>\tau\end{cases}
$$

with $\tau$ being sufficiently small, we get

$$
W\left[\mathbf{w}^{*}(\cdot)\right]=\frac{M J_{o}^{2}}{2}-\mu(\tau)
$$

where $\lim _{\tau \rightarrow o} \mu(\tau)=0$. Hence, we have

$$
\lim _{\tau \rightarrow o} W\left[\mathbf{w}^{*}(\cdot)\right]=W^{+} .
$$

And equality (8) holds.

Now, let us discuss an example of a mechanical system for which (8) is true. Let us consider a one-dimensional chain of $N$ interconnected point masses connected to the base by elastic-dissipative links. Such a system can be used for modeling the dynamics of different real structures, for example, a motion of railway carriages following a locomotive, or a horizontal displacement of a multistory building under seismic displacement of its base, etc. The equations of motion can be expressed in the form

$$
\begin{aligned}
m_{1}\left(\ddot{x}_{1}+w\right)= & -\varphi_{1}\left(x_{1}\right)-g_{1}\left(\dot{x}_{1}\right)+\varphi_{2}\left(x_{2}-x_{1}\right) \\
& +g_{2}\left(\dot{x}_{2}-\dot{x}_{1}\right) \\
. & \cdot \\
m_{i}\left(\ddot{x}_{i}+w\right)= & -\varphi_{i}\left(x_{i}-x_{i-1}\right)-g_{i}\left(\dot{x}_{i}-\dot{x}_{i-1}\right) \\
& +\varphi_{i+1}\left(x_{i+1}-x_{i}\right)+g_{i+1}\left(\dot{x}_{i+1}-\dot{x}_{i}\right)
\end{aligned}
$$

$m_{n}\left(\ddot{x}_{n}+w\right)=-\varphi_{n}\left(x_{n}-x_{n-1}\right)-g_{n}\left(\dot{x}_{n}-\dot{x}_{n-1}\right)$

and initial conditions may be taken as

$$
x_{i}(0)=0, \quad \dot{x}_{i}(0)=0 \quad i \in[1, n]
$$

The scalar function $w=w(t)$ is supposed to describe the acceleration of the base and satisfies condition (4); and the functions $g_{i}, \varphi_{i}$, describing the elastic-dissipative links of the system are continuous and meet the condition

$$
g_{i}(\eta) \operatorname{sign}(\eta) \geq 0, \quad \varphi_{i}(\eta) \operatorname{sign}(\eta) \geq 0
$$

In this case, the power of the dissipative forces is equal to

$$
-\sum_{i=1}^{n-1} g_{i+1}\left(\dot{x}_{i}-\dot{x}_{i+1}\right)\left(\dot{x}_{i}-\dot{x}_{i+1}\right)-g_{1}\left(\dot{x}_{1}\right) \dot{x}_{1} \leq 0
$$

and the potential energy is

$$
\prod=\sum_{i=1}^{n-1} \int_{o}^{\left\langle x_{i+1}-x_{i}\right\rangle} \varphi_{i+1}(\eta) d \eta+\int_{o}^{x_{i}} \varphi_{1}(\eta) d \eta \geq 0
$$

with $\Pi$ vanishing $(\Pi=0)$ when all $x_{i}=0$. Thus, conditions (2), (5), and (11) are satisfied. If the base is subjected to an instantaneous impact so that

$$
w(t)=J_{o} \delta(t)
$$

then from the equations of motion (14) we immediately obtain the instantaneous increment in the velocity $\dot{x}_{i}(0)$ :

$$
\dot{x}_{i}(0)=-J_{o} \quad i \in[1, n]
$$

Hence, condition (12) is also valid.

Thus, the disturbance that maximizes the sum of the kinetic and potential energies of the given mechanical system is an instantaneous impact of intensity $J_{o}$ [equality (8) being true], and the desired value of the maximum response is given by

$$
W^{+}=\frac{M J_{o}^{2}}{2} \quad\left(M=\sum_{i=1}^{n} m_{i}\right)
$$

Note here that the value $W^{+}$also enables us to estimate a maximal displacement in system (14) described by

$$
\sigma_{o}[w(\cdot)]=\max \left\{\sigma_{1}, \sigma_{2}, \ldots, \varphi_{n}\right),
$$

where $\sigma_{1}=\sup _{t \in[0, \infty)}\left|x_{1}[t, w(\cdot)]\right|$,

$$
\begin{aligned}
\sigma_{i+1}=\sup _{t \in[0, \infty)}\left|x_{i}[t, w(\cdot)]-x_{i+1}[t, w(\cdot)]\right| & \\
& i \in[1, n-1],
\end{aligned}
$$

and $\left\{x_{1}[t, w(\cdot)], \ldots ., x_{i}[t, w(\cdot)], \ldots\right.$, $\left.x_{n}[t, w(\cdot)]\right\}$ is a solution of system (14) corresponding to the disturbance $w(t)$.

Indeed, from $\Pi \leq W^{+}$, taking into account the 
second relationship in (15), we arrive at the inequality

$$
\sigma_{i} \leq\left|s_{i}^{*}\right|, \quad i \in[1, n],
$$

where $s_{i}^{*}$ is the maximum (in absolute value) root of the equation

$$
\int_{o}^{s_{i}^{*}} \varphi_{i}(\eta) d \eta=W^{+}
$$

This means that the desired estimate of the maximal displacement is given by

$$
\sigma_{o}[w(\cdot)] \leq \max \left(\left|s_{i}^{*}\right|,\left|s_{2}^{*}\right|, \ldots,\left|s_{n}^{*}\right|\right)=S^{+} .
$$

It is worth mentioning that the estimate may appear to be significantly overstated in the sense that the value

$$
\sigma^{+}=\sup _{w(\cdot) \in \Theta} \sigma_{o}[w(\cdot)],
$$

will be drastically less than $S^{+}$. However, if (14) is a single-mass system, then the value of $\sigma^{+}$is the same as the estimate $S^{+}$, provided the dissipative link is absent.

\section{APPLICATION OF RESULTS}

Now let us discuss an example of results above to estimate exactly the maximum displacement of a second-order nonlinear system with damping.

Let us consider the following system

$$
\begin{gathered}
\dot{x}=y, \quad \dot{y}=-g(x, y)-\varphi(x)+u(t), \\
x(0)=y(0)=0 .
\end{gathered}
$$

For the continuous functions $g$ and $\varphi$, it is supposed that

$$
\begin{gathered}
g(x, y) \operatorname{sign}(y) \geq 0, \\
\frac{g\left(x, y_{1}\right)}{y_{1}} \geq \frac{g\left(x, y_{2}\right)}{y_{2}} \text { at }\left|y_{2}\right|>\left|y_{1}\right|>0, \\
\varphi(x) \operatorname{sign}(x) \geq 0, \quad \varphi(x) \neq \equiv 0, \\
\varphi\left(x_{1}\right) \geq \varphi\left(x_{2}\right) \text { at } \quad x_{1}>x_{2} .
\end{gathered}
$$

Depending upon the situation, by the external disturbance $u(t)$, we understand $w(t)$ from the class $\Theta$, or some instantaneous impact

$$
\Delta^{+}(t)=J_{o} \delta(t) \quad\left[\text { or } \quad \Delta^{-}(t)=-J_{o} \delta(t)\right] .
$$

Note that if $u(t)=w(t)$, then, with (18) and (19) taken into consideration, the following estimate is valid

$$
W[w(\cdot)] \leq J_{o}^{2} / 2,
$$

where $W[w(\cdot)]$ is maximum of the sum of kinetic and potential energy

$$
E=\frac{y^{2}}{2}+\int_{o}^{x} \varphi(x) d x
$$

calculated for the solution $\{x[t, w(\cdot)], y[t, w(\cdot)]\}$ of system (17).

Let us state the following problem: find the value $\sigma^{+}$determined in accordance with (16), provided the maximum displacement given by

$$
\sigma_{o}[w(\cdot)]=\sup _{t \in[o, \infty)}|x[t, w(\cdot)]| .
$$

Let us first consider the motion of system (17) under instantaneous impact (20). This motion is described as

$$
\begin{gathered}
\dot{x}=y, \quad \dot{y}=-g(x, y)-\varphi(x) \\
x(0)=0, \quad y(0)=J_{o} \\
{\left[y(0)=-J_{o}, \quad \text { if } u(t)=\Delta^{-}(t)\right] .}
\end{gathered}
$$

Note here some features of system (22). Let us separate the initial segments of the system trajectories corresponding to the disturbances $\Delta^{+}(t)$ and $\Delta^{-}(t)$ and lying in the first and third quadrants of the phase plane $\{x, y\}$, respectively. Denote these segments by $F^{+}$and $F^{-}$(Fig. 1).

The curve $F^{+}$is described as follows

$$
y=y_{o}^{+}(x),
$$

and the curve $F^{-}$is given by

$$
y=y_{o}^{-}(x) .
$$

Now using the assumed properties of the functions $g, \varphi$ it is easy to show that a) unique roots of the equations

$$
y_{o}^{+}(x)=0 \quad \text { and } \quad y_{o}^{-}(x)=0
$$

denoted by $\lambda^{+}>0, \lambda^{-}<0$ assume finite values; and $b$ ) 


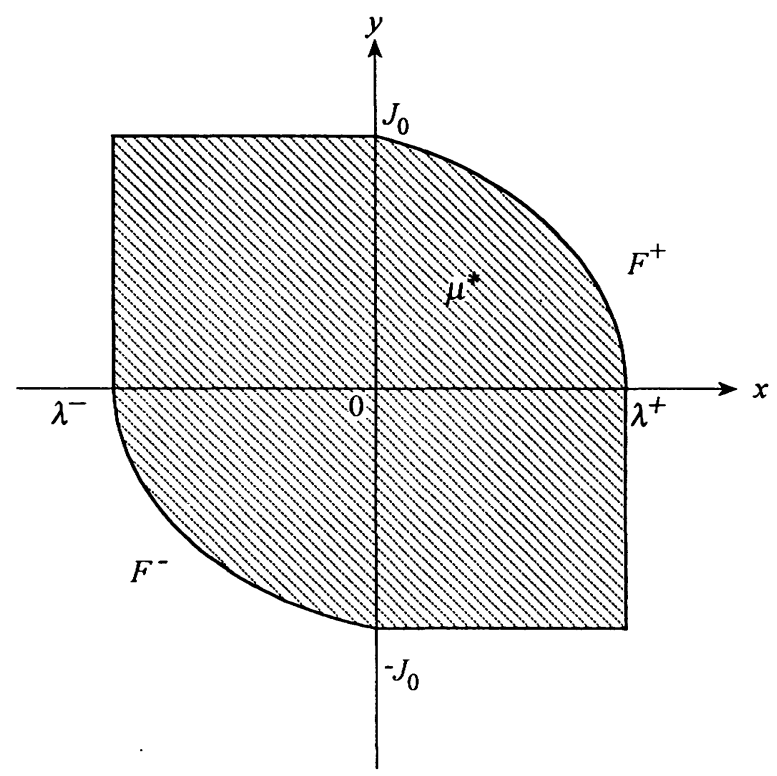

FIGURE 1

$$
\max \left\{\sigma_{o}\left[\Delta^{+}(\cdot)\right], \sigma_{o}\left[\Delta^{-}(\cdot)\right]\right\} \equiv \max \left\{\lambda^{+},\left|\lambda^{-}\right|\right\} .
$$

On the phase plane, let us determine a set of points $\mu^{*}$ containing the point $\{0,0\}$ and bounded by the curves $F^{+}$and $F^{-}$within the first and the third quadrants, and by rectangles with sides $\left\{\left|\lambda^{-}\right|, J_{o}\right\}$ and $\left\{\lambda^{+}, J_{o}\right\}$, within the second and the fourth (Fig. 1). Let us show that a phase trajectory of system (17), corresponding to an arbitrary disturbance $w(t)$ of the class $\Theta$, will not leave the set $\mu^{*}$.

Now, let us have a look at the phase plane $\{x$, $y$ \} of system (17). According to (17) and with (18) and (19), the phase trajectory [that corresponds to an arbitrary disturbance $w(t)]$ in the first quadrant connects a point on the $y$-axis (or $x$-axis) to a point on the $x$-axis. Now, let us separate the phase trajectory segments fully lying within the first quadrant and denote them by $F_{i}$. The segments are numbered according to the time they pass the first quadrant. Let $t_{i}^{-}$and $t_{i}^{+}$be the instants at which motion starts and ends along the segment $F_{i}$; and let $x=x[t, w(\cdot)], y=y[t, w(\cdot)]$ for $t \in\left[t_{i}^{-}, t_{i}^{+}\right]$be a parametric equation for the segment $F_{i}$. We denote $x_{i}^{-}=x\left[t_{i}^{-}, w(\cdot)\right], x_{i}^{+}=$ $x\left[t_{i}^{+}, w(\cdot)\right], y_{i}^{-}=y\left[t_{i}^{-}, w(\cdot)\right], y_{i}^{+}=y\left[t_{i}^{+}, w(\cdot)\right]$. According to the definition of segments $F_{i}$, we have $y_{i}^{+}=0$. Because $y=y[t, w(\cdot)]>0$ for $t \in$ $\left(t_{i}^{-}, t_{i}^{+}\right), y$ may be expressed through $x: y=y_{i}(x)$ at $x \in\left[x_{i}^{-}, x_{i}^{+}\right]$. Among all $F_{i}$, we then choose those starting at the points lying on the $y$-axis. Denote a subset of all such $F_{i}$ through $\Gamma_{o}$, that is,
$F_{i} \in \Gamma_{o}$, if $y_{i}^{-} \geq 0, x_{i}^{-}=0$. Note that $\Gamma_{o}$ is nonempty if the set of all $F_{i}$ is non-empty, because under zero initial conditions in system (17) $F_{i}$ [with allowance for (18), (19)] necessarily belongs to $\Gamma_{o}$. Consider two segments, $F_{j}$ and $F_{k}$, so that these segments belong to $\Gamma_{o}$, where $F_{i}$ whose indices range from $j+1$ through $k-1$ do not belong to $\Gamma_{\mathrm{o}}$. Now let us make some auxiliary constructions. We consider the following two possible variants for mutual position of segments $F_{j+1}$ and $F_{j}:$ 1) $F_{j+1}$ and $F_{j}$ have at least one common point; 2) $F_{j+1}$ and $F_{j}$ have no common points.

Let us consider the first variant, and denote by $\left\{x_{j+1}^{j}, y_{j+1}^{j}\right\}$ the first (after the moment $t_{j+i}^{-}$) common point. Let us construct a curve composed from a piece of the segment $F_{j}$ (from the start up to the intersection point) and a piece of the segment $F_{j+1}$ (from the intersection point up to the end) (Fig. 2). This curve, denoted through $H_{j}^{1}$, may be written as

$$
y=y_{j}^{i}(x)=\left\{\begin{array}{ll}
y_{j}(x), & x \in\left[0, x_{j+1}^{j}\right] \\
y_{j+1}(x), & x \in\left[x_{j+1}^{j}, x_{j+1}^{+}\right]
\end{array} .\right.
$$

Denote by $\xi_{j+1}^{j}$ and $\zeta_{j+1}^{j}$ the instants when the phase trajectory segments $F_{j+1}$ and $F_{j}$ pass through the point $\left\{x_{j+1}^{j}, y_{j+1}^{j}\right\}$ :

$$
\begin{gathered}
\xi_{j+1}^{j} \in\left[t_{j}^{-}, t_{j}^{+}\right], \quad \zeta_{j+1}^{j} \in\left[t^{-}{ }_{j+1}, t_{j+1}^{+}\right], \\
x\left[\xi_{j+1}^{j}, w(\cdot)\right]=x\left[\zeta_{j+1}^{j}, w(\cdot)\right]=x_{j+1}^{j}, \\
y\left[\xi_{j+1}^{j}, w(\cdot)\right]=y\left[\zeta_{j+1}^{j}, w(\cdot)\right]=y_{j+1}^{j} .
\end{gathered}
$$

Now let the second variant take place, that is, $F_{j+1}$ and $F_{j}$ do not intersect. Then, according to (17) and with (18) and (19), the segments $F_{j+1}$ lie between $F_{j}$ and the $x$-axis:

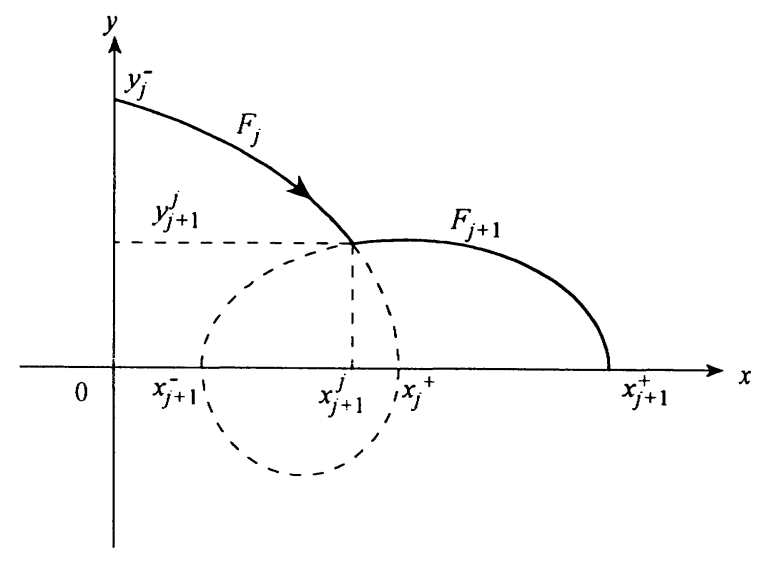

FIGURE 2 


$$
0<y_{j+1}(x)<y_{j}(x) \text { at } x \in\left(x_{j+1}^{-}, x_{j+1}^{+}\right) .
$$

In this case, the curve $H_{j}^{i}$ is assumed to coincide with $F_{j}$ :

$$
y_{j}^{\prime}(x)=y_{j}(x)
$$

Suppose that the curve $H_{j}^{r}$, where $r=l-j$ and $l<k-1$, has been built, and now we will show how to construct the curve $\boldsymbol{H}_{j}^{r+1}$. As before we have two variants of mutual positions of $\boldsymbol{H}_{j}^{r}$ and $F_{l+1}:$ 1) $H_{j}^{r}$ and $F_{l+1}$ intersect: 2) $H_{j}^{r}$ and $F_{l+1}$ have no common points. If the first variant takes place, then denote by $\left\{x_{l+1}^{n}, y_{l+1}^{n}\right\}$ the first (after $\left.t_{l+1}^{-}\right)$intersection point of $\boldsymbol{H}_{j}^{r}$ and $\boldsymbol{F}_{l+1}$. Here the superscript index $n$ denotes the number of the segment $F_{n}$ that belongs to the curve $H_{j}^{r}$, and is intersected by the segment $F_{l+1}$; in this case $n \in$ $[j, l]$. If an intersection point falls into the "joint" of the segments $F_{m}$ and $F_{n}$, then the smaller of the numbers $m$ and $n$ is taken as a superscript. Let us construct the curve $H_{j}^{r+1}$ composed of the piece of the curve $H_{j}^{r}$ (from the start up to the intersection point) and the piece of segment $F_{l+1}$ (from the intersection point up to the end). The curve $H_{j}^{r+1}$ is given by

$$
y=y_{j}^{r+1}(x)=\left\{\begin{array}{ll}
y_{j}^{r}(x), & x \in\left[0, x_{l+1}^{n}\right] \\
y_{l+1}(x), & x \in\left[x_{l+1}^{n}, x_{l+1}^{+}\right]
\end{array} .\right.
$$

Denote also by $\xi_{l+1}^{n}, \zeta_{l+1}^{n}$ the instants when the curve segments $H_{j}^{r}$ and $F_{l+1}$ pass through point $\left\{x_{l+1}^{n}, y_{l+1}^{n}\right\} ; \xi_{l+1}^{n} \in\left[t_{n}^{-}, t_{n}^{+}\right], \zeta_{l+1}^{n} \in\left[t_{l+1}^{-}, t_{l+1}^{+}\right]$. If the second variant takes place, that is, $H_{j}^{r}$ and $F_{l+1}$ do not intersect, then the segment $F_{l+1}$ lies below the curve $H_{j}^{r}$. In this case $H_{j}^{r+1}$ is assumed to coincide with $H_{j}^{r}$ :

$$
y_{j}^{r+1}(x)=y_{j}^{r}(x) .
$$

Expression (25) combined with (23), (24), and (26), constitutes a recursive formula to determine the curve $\boldsymbol{H}_{j}^{p}$ for $p \in[1, k-1]$. To all curves $\boldsymbol{H}_{j}^{p}$ let us add the curves $F_{j}$ from the set $\Gamma_{o}$ denoting them by $H_{j}^{o}: y_{j}^{o}(x)=y_{j}(x)$. The set of curves thus obtained is denoted by $G_{o}$. For each $H_{j}^{p} \in G_{o}$, we also determine a set of points within the phase plane; this set contains the point $\{0,0\}$ and is bounded by the coordinate axes and the curve $H_{j}^{p}$. Denote this set by $\theta_{j}^{p}$ (Fig. 3). According to the definition of the set $G_{o}$, for every segment of the phase trajectory $F_{i}$ there can be found a curve $H_{j}^{p}$ such that $F_{i}$ does not leave the set $\theta_{j}^{p}$.

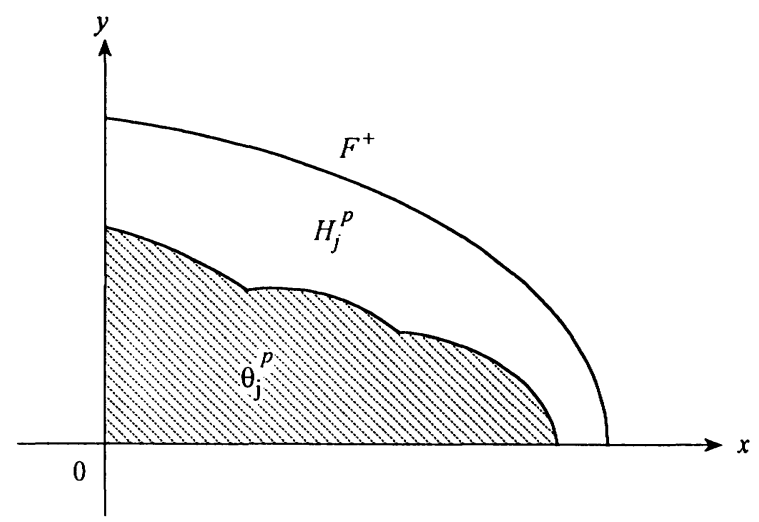

FIGURE 3

Thus, to prove that the system phase trajectory (17) lies within the first quadrant of the phase plane and does not leave the set $\mu^{*}$, it is sufficient to prove that the curves $H_{j}^{p} \in G_{o}$ do not intersect $F^{+}$and do not leave the set $\mu^{*}$. Let us examine the curve $\boldsymbol{H}_{j}^{p}$. For simplicity, assume that this curve consists of only two pieces of the segments $F_{j}$ and $F_{n}$. Let the coordinates of the intersection point of $F_{j}$ and $F_{n}$ be $\left\{x_{n}^{j}, y_{n}^{j}\right\}$; and the instants when the segments $F_{j}$ and $F_{n}$ pass through this point be denoted by $\xi_{n}^{j}, \zeta_{n}^{j}\left(\xi_{n}^{j}<\zeta_{n}^{j}\right)$, respectively. Consider an external disturbance in the form

$$
w^{*}(t)=\left\{\begin{array}{ll}
w(t), & t \in\left[0, \xi_{n}^{j}\right) \\
w\left(t-\xi_{n}^{j}+\zeta_{n}^{j}\right), & t \geq \xi_{n}^{j}
\end{array} .\right.
$$

Note that if $w(t)$ belongs to the class $\Theta$, the function $w^{*}(t)$ will also belong to it. Moreover, the phase trajectory $\{x[t, w(\cdot)], y[t, w(\cdot)]\}$ corresponding to the disturbance $w(t)$, differs from the phase trajectory $\left\{x\left[t, w^{*}(\cdot)\right], y\left[t, w^{*}(\cdot)\right]\right\}$ [corresponding to the disturbance $\left.w^{*}(t)\right]$ only within the interval $\left(\xi_{n}^{j}, \zeta_{n}^{j}\right)$. Within the time interval $\left[t_{j}^{-}\right.$, $\left.t_{n}^{+}+\xi_{n}^{j}-\zeta_{n}^{j}\right]$, the phase trajectory $\left\{x\left[t, w^{*}(\cdot)\right]\right.$, $\left.y\left[t, w^{*}(\cdot)\right]\right\}$ will coincide with the curve $H_{j}^{p}$. Because $y\left[t, w^{*}(\cdot)\right]>0$ in the interval $\left(t_{j}^{-}, t_{n}^{+}+\xi_{n}^{j}\right.$ $\left.-\zeta_{n}^{j}\right), y$ and $t$ can be expressed as a function of $x$ :

$$
y=y_{j}^{p}(x), \quad t=t_{j}^{p}(x) \quad \text { at } \quad x \in\left[x_{j}^{-}, x_{n}^{+}\right] .
$$

We can also find the function $w_{j}^{p}(x)=w^{*}\left[t_{j}^{p}(x)\right]$ for $x \in\left[x_{j}^{-}, x_{n}^{+}\right]$. The function $y_{j}^{p}(x)$ for $x \in\left[x_{j}^{-}\right.$, $\left.x_{n}^{+}\right]$satisfies the differential equation

$$
\frac{d y}{d x}=\frac{-g(x, y)-\varphi(x)+w_{j}^{p}(x)}{y}, \quad y\left(x_{j}^{-}\right)=y_{j}^{-}
$$


derived from system (17) by dividing the second equation by the first one. Let us write Eq. (27) in the integral form:

$y_{j}^{p}(x)=y_{j}^{-}-\int_{x_{j}^{-}}^{x} R\left[x, y_{j}^{p}(x)\right] d x+\int_{x_{j}^{-}}^{x} \frac{w_{j}^{p}(x)}{y_{j}^{p}(x)} d x$,

where

$$
R\left[x, y_{j}^{p}(x)\right]=\frac{g\left[x, y_{j}^{p}(x)\right]+\varphi(x)}{y_{j}^{p}(x)} .
$$

Note that according to the definition of the curve $H_{j}^{p}$, we obtain $x_{j}^{-}=0$ and

$$
\int_{0}^{x} \frac{w_{j}^{p}(x)}{y_{j}^{p}(x)} d x=\int_{t_{j}^{-}}^{t_{j}^{p}(x)} w^{*}(t) d t \leq \int_{t^{-}}^{\infty}|w(t)| d t .
$$

The function $y=y_{o}^{+}(x)$ describing the curve $F^{+}$ satisfies the differential equation

$$
\frac{d y}{d x}=\frac{-g(x, y)-\varphi(x)}{y}, \quad y(0)=J_{o}
$$

for $x \in\left[0, \lambda^{+}\right]$. We can write this equation in the integral form:

$$
y_{o}^{+}(x)=J_{o}-\int_{0}^{x} R\left[x, y_{o}^{+}(x)\right] d x .
$$

Subtracting expression (28) from both sides of this equation we obtain:

$$
\begin{aligned}
y_{o}^{+}(x) & -y_{j}^{p}(x)=J_{o}-y_{j}^{-}-\int_{0}^{x} \frac{w_{j}^{p}(x)}{y_{j}^{p}(x)} d x \\
& -\int_{0}^{x} \eta\left[x, y_{j}^{p}(x)\right] d x,
\end{aligned}
$$

where $\eta\left[x, y_{j}^{p}(x)\right]=R\left[x, y_{o}^{+}(x)\right]-R\left[x, y_{j}^{p}(x)\right]$. Let us show that the curve $H_{j}^{p}$ does not intersect the curve $F^{+}$and hence does not leave the set $\mu^{*}$. This is proved by contradiction. Suppose that the curve $H_{j}^{p}$ intersects the curve $F^{+}$at some point. In other words, there exists $x^{*}$ such that

$y_{o}^{+}\left(x^{*}\right)=y_{j}^{p}\left(x^{*}\right), \quad y_{o}^{+}(x)>y_{j}^{p}(x)$ at $x \in\left[0, x^{*}\right)$.

Taking into account the second inequality (18), we arrive at

$$
\eta\left[x, y_{j}^{p}(x)\right]<0 \quad \text { at } \quad x \in\left[0, x^{*}\right) .
$$

Now consider the difference

$$
K_{j}^{p}(x)=J_{o}-y_{j}^{-}-\int_{0}^{x} \frac{w_{j}^{p}(x)}{y_{j}^{p}(x)} d x
$$

and use estimate (21) for the maximal energy of system (17). Let

$$
I_{o} \int_{0}^{t_{j}^{-}}|w(t)| d t
$$

If we consider disturbances $w^{\prime}(t)$ of the form

$$
w^{\prime}(t)= \begin{cases}w(t), & t \in\left[0, t_{j}^{-}\right), \\ 0, & t \geq t_{j}^{-}\end{cases}
$$

then according to estimate (21) we have

$$
W\left[w^{\prime}(\cdot)\right] \leq I_{o}^{2} / 2
$$

and, given the expression for the energy $E$, we get

$$
y_{j}^{-} \leq I_{o} .
$$

Thus, taking (29), into account, we have

$$
\begin{aligned}
K_{j}^{p}(x) & \geq J_{o}-I_{o}-\int_{0}^{x^{*}} \frac{w_{j}^{p}(x)}{y_{j}^{p}(x)} d x \\
& \geq J_{o}-I_{o}-\int_{t_{j}^{-}}^{\infty}|w(t)| d t \geq 0 .
\end{aligned}
$$

From relationship (30), with (31), we immediately reach the contradiction

$$
y_{o}^{+}\left(x^{*}\right)-y_{j}^{p}\left(x^{*}\right)>0 .
$$

Hence, the curve $H_{j}^{p}$ does not intersect $F^{+}$and does not leave the set $\mu^{*}$. A similar proof may be applied to any curve $H_{j}^{p}$ from the set $G_{o}$. So we have shown that the system phase trajectory (17) lying within the first quadrant of the phase plane does not leave the set $\mu^{*}$. This means that for all $x_{i}^{+}$, the inequality $x_{i}^{+}<\lambda^{+}$is valid. Then, according to (17), (18), and (19), for any trajectory segment within the fourth quadrant, the inequality $x[t, w(\cdot)]<\lambda^{+}$holds. In addition, because of estimate (21) in the fourth quadrant, the inequality $|y[t, w(\cdot)]| \leq J_{o}$ holds. Hence, the phase trajectory lying within the fourth quadrant does not leave the set $\mu^{*}$.

The behavior of the phase trajectory within the third and second quadrants is analyzed, with al- 
lowance made for the properties of the functions $g$ and $\varphi$ [see (18), (19)], in a similar way, if in Eqs. (17) the change of variables $x^{\prime}=-x, y^{\prime}=-y$ is made.

Thus, the phase trajectory corresponding to any disturbance $w(t)$ of the class $\Theta$, does not leave the set $\mu^{*}$. This means that for the maximal displacement in (17) the estimate

$$
\sigma_{o}[w(\cdot)] \leq \max \left\{\lambda^{+},\left|\lambda^{-}\right|\right\} \quad \forall w(\cdot) \in \Theta
$$

holds. To prove the equality

$$
\sigma^{+}=\max \left\{\lambda^{+},\left|\lambda^{-}\right|\right\}
$$

it is sufficient to consider the disturbances

$$
w_{+}^{*}(t)=+J_{o} \nu(t), \quad w_{-}^{*}(t)+-J_{o} \nu(t),
$$

where $\nu(t)$ is the function of the kind (13). As $\tau$ tends to zero, we obtain

$$
\begin{aligned}
& \max \left\{\lim _{\tau \rightarrow o} \sigma_{o}\left[w_{+}^{*}(\cdot)\right],\right. \\
& \left.\qquad \lim _{\tau \rightarrow o} \sigma_{o}\left[w^{*}(\cdot)\right]\right\}=\max \left\{\lambda^{+},\left|\lambda^{-}\right|\right\} .
\end{aligned}
$$

Hence, the instantaneous impact of intensity $J_{o}$ or $-J_{o}$ results in the maximum value $\sigma^{+}$of the maximal displacement $\sigma_{o}[w(\cdot)]$ in (17). This result generalizes the results obtained by Balandin [1989].

\section{EXAMPLE}

As an example let us consider a nonlinear oscillator described by the equation

$$
\ddot{x}=-q \dot{x}+k|x| x+w(t), \quad \dot{x}(0)=x(0)=0 .
$$

We assume that $q=0.528$ and $k=0.441$. Having solved this differential equation numerically, we can compare the above estimate $\sigma^{+}$with the values of the maximal displacement $\sigma_{o}[w(\cdot)]$ under various disturbances $w(t)$. Let us consider shock disturbances of two kinds

$$
\begin{aligned}
& w_{1}(t)=\left\{\begin{array}{lr}
1 / T, & 0 \leq t \leq T \\
0, & t>T
\end{array}\right. \\
& w_{2}(t)=\left\{\begin{array}{lr}
6 t(T-t) / T^{3}, & 0 \leq t \leq T \\
0, & t>T
\end{array}\right.
\end{aligned}
$$

Table 1. Maximal Displacement Values

\begin{tabular}{llllll}
\hline$T$ & 0.20 & 0.50 & 1.00 & 1.50 & 1.80 \\
$\sigma_{1}$ & 0.998 & 0.997 & 0.996 & 0.990 & 0.985 \\
$\sigma_{2}$ & 0.999 & 0.999 & 0.998 & 0.995 & 0.992 \\
\hline
\end{tabular}

for which the equality

$$
\int_{o}^{\infty} w_{1}(t) d t=\int_{o}^{\infty} w_{2}(t) d t=1
$$

holds. If we let $\Theta_{o}$ be the class of disturbances consisting of $w_{1}(t)$ and $w_{2}(t)$, then for this class the greatest value of the maximal displacement $\sigma^{+}$will be equal to 1.000 . Table 1 contains maximal displacement values for the oscillator influenced by the disturbances from the class $\Theta_{o}$. The displacements depend on the duration of the disturbance. The values of $\sigma_{1}$ and $\sigma_{2}$ differ from $\sigma_{+}$ at most by $1.5 \%$. Hence, the magnitude $\sigma^{+}$can be considered a sufficiently good estimate for the maximal displacement of the given system under forces belonging to the class $\Theta_{o}$.

\section{SUMMARY}

In this paper, an estimate for the maximal energy is obtained for mechanical systems under disturbances the integral of whose absolute value is below a given value $J_{o}$. The conditions for this estimate to be exact are formulated. An example is given that shows how the results obtained can be used for determining the maximal displacement in a second-order nonlinear system. These results may also be used for estimating a guaranteed quality of various mechanical systems, in particular shock isolation systems.

\section{REFERENCES}

Balandin, D. V., 1989, "Optimization of Shock Absorbers for a Class of External Loadings," Izv. AN SSSR. M.T.T. (News of the USSR Academy of Sciences, Mechanics of Solids), Vol. 24, pp. 53-60 (In Russian).

Bolotnik, N. N., 1976, "The Problems of Optimal Shock Isolation for Classes of External Actions," Izv. AN SSSR. M.T.T. (News of the USSR Academy of Sciences, Mechanics of Solids). Vol. 11, pp. 34-41 (In Russian). 
Boulgakov, B. V., 1946. "About Accumulating the Disturbances in Linear Oscillating Systems with Constant Parameters,' DAN SSSR (Reports of the USSR Academy of Sciences), Vol. 51, pp. 339-342 (In Russian).
Pars, L. A., 1968, A Treatise on Analytical Dynamics, John Wiley \& Sons, Inc., New York.

Sevin, E., 1957, "Min-Max Solutions for the Linear Mass-Spring System," ASME Journal of Applied Mechanics, Vol. 24, pp. 131-136. 

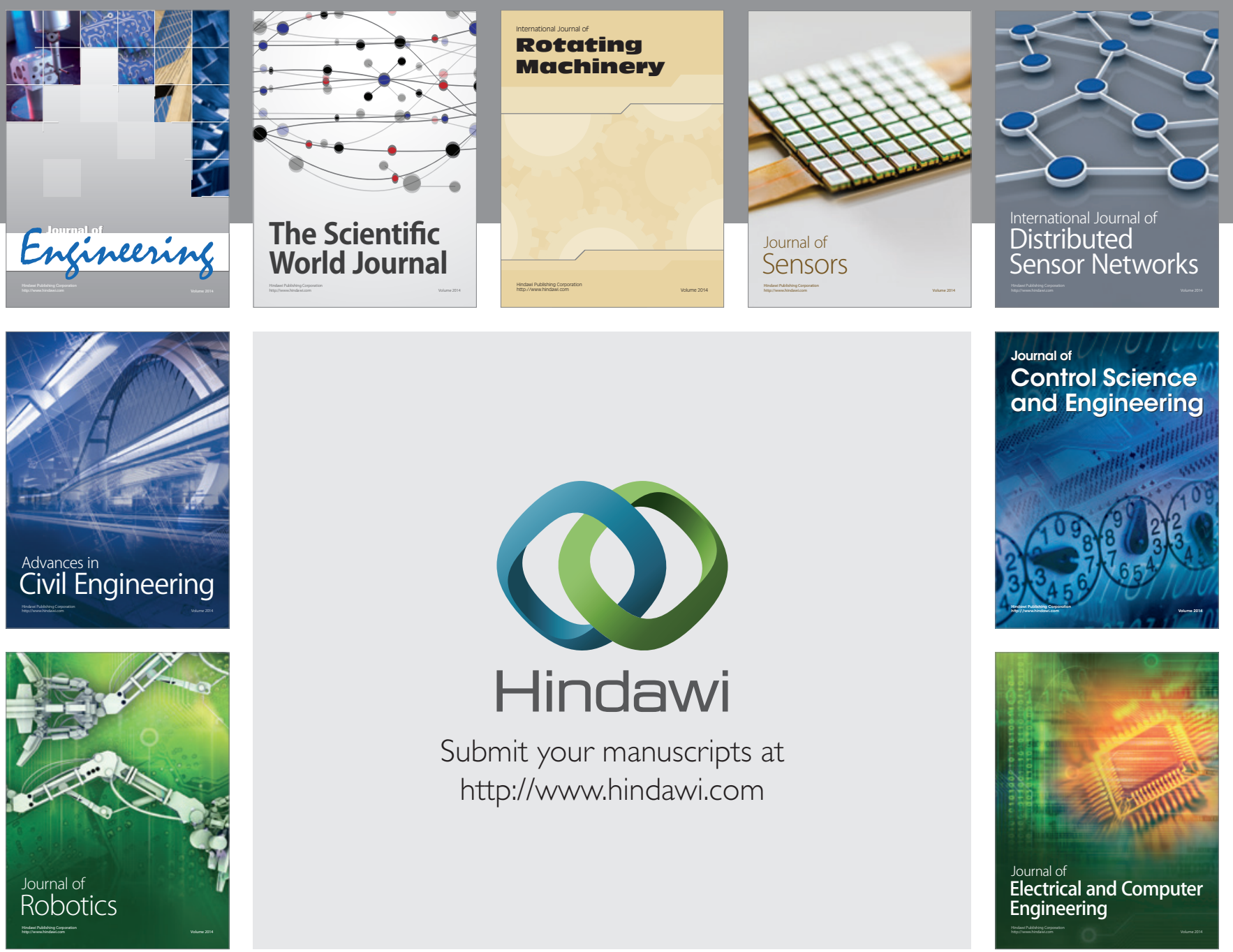

Submit your manuscripts at

http://www.hindawi.com
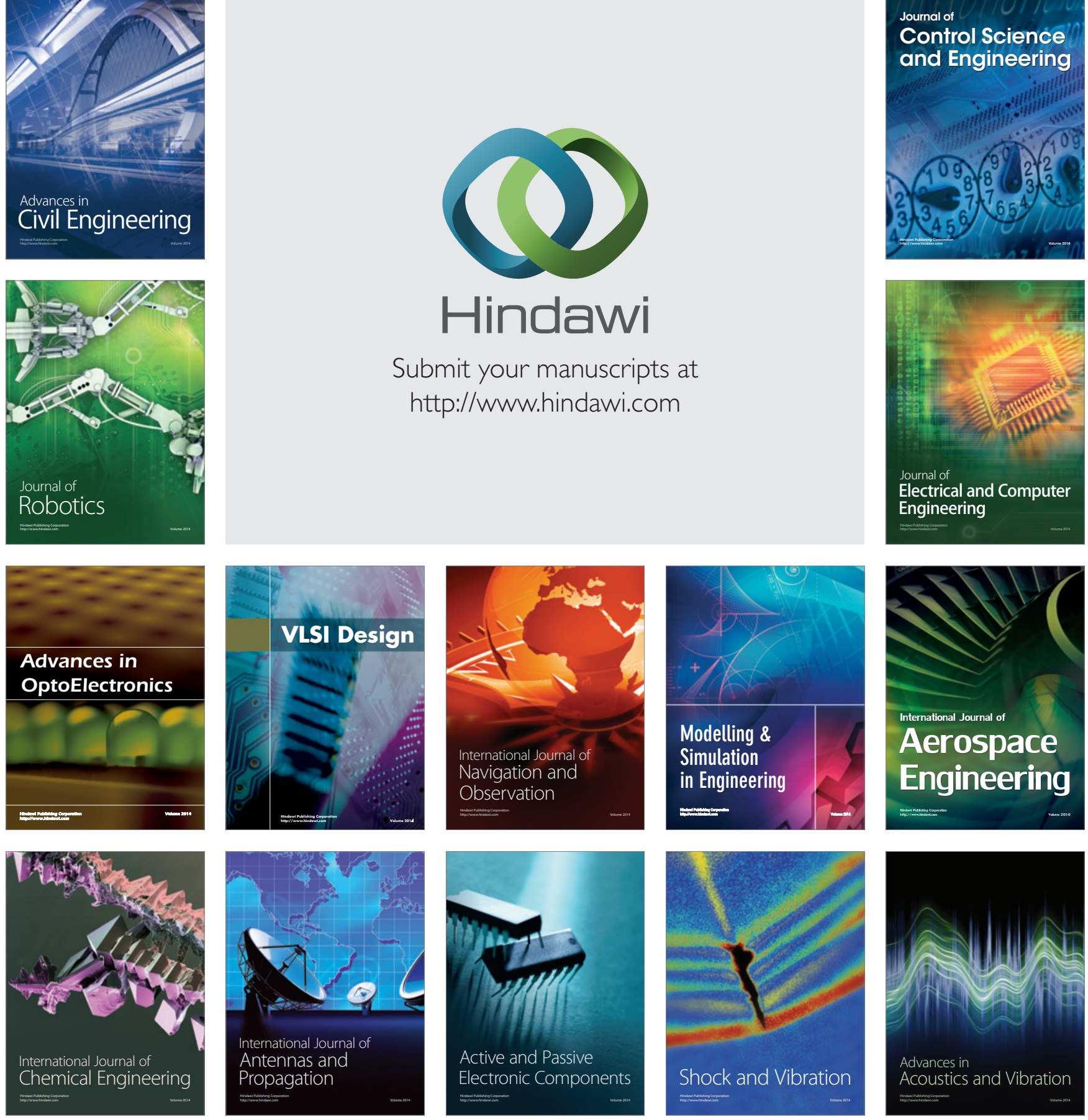